\title{
Laminar flow of a gas in a tube with large temperature differences
}

\author{
F. J. Higuera \\ ETSI Aeronáuticos, UPM, Plaza Cardenal Cisneros 3, 28040 Madrid, Spain
}

\begin{abstract}
The laminar low Mach number flow of a gas in a tube is analyzed for very small and very large values of the inlet-to-wall temperature ratio. When this ratio tends to zero, pressure forces confine the cold gas to a thin core around the axis of the tube. This core is neatly bounded by an ablation front that consumes it at a finite distance from the tube inlet. When the temperature ratio tends to infinity, the temperature of the gas increases smoothly from the wall to the axis of the tube and the shear stress and heat flux are positive at the wall despite the fact that the viscosity and thermal conductivity of the gas scaled with their inlet values tend to zero at the wall.
\end{abstract}

\section{INTRODUCTION}

The laminar low Mach number flow of a gas in a tube and the heat transfer between the gas and the tube wall are classical problems that have been investigated extensively using experimental, numerical, and approximate analytical methods to take into account the variations of the gas density, viscosity, and thermal conductivity with temperature. ${ }^{1-4}$ Correlations for the friction coefficient and the Nusselt number have been obtained which show that the first quantity is more sensitive than the second to temperature variations of the gas properties, ${ }^{4}$ and the effect of the radial velocity induced by the variation of the gas density has been quantified. ${ }^{2,3}$ It has been found that most of the effects of variable gas properties can be approximately taken into account through fairly simple correction factors for the friction coefficient and the Nusselt number evaluated for constant fluid properties, and thus cases in which a full solution of the conservation equations for the gas needs to be computed are rare. This is fortunate for many engineering applications, but the question remains of what is the structure of the flow for very small and very large values of the inlet-to-wall temperature ratio, when the gas properties change much across the tube and the correction factors approximation is not adequate.

The question has been addressed for the related cases of a boundary layer of a gas on a solid surface kept at a temperature much smaller or much larger than the temperature of the gas stream, and of a gas jet discharging into an infinite expanse of the same gas at a different temperature, both of which are elements of the flow in ejectors, combustion chambers, chemical reactors, and propulsion and plasma devices. In a jet diffusion flame, for example, combustion leads to an annular layer of very hot gas surrounding the flame, which separates an inner cold fuel jet from an outer, stagnant or coflowing, cold oxidizer. Numerical computations and analyses of the reactive flow in the near wake of an injector, ${ }^{5}$ the interactive flow in the boundary layer around a step elevation of surface temperature, ${ }^{6}$ and discharging laminar gas jets, ${ }^{7-10}$ all reveal the existence of neatly defined boundaries between cold and hot gases, which in the limit when the cold-to-hot gas temperature ratio tends to zero become ablation fronts where the heat flux tends to zero and a mass flux crosses from the cold to the hot gas side. The work of Sánchez-Sanz et al. ${ }^{7-10}$ points out the similarity between these ablation fronts and the fronts found by Zeldovich and Kompaneetz ${ }^{11}$ in heated solids with temperaturedependent conductivity and by Williams, ${ }^{12}$ Tarifa et al. ${ }^{13}$ and Crespo and Liñán ${ }^{14}$ in the heating and vaporization of gas pockets and liquid drops, respectively.

Some of the applications mentioned above featuring gas jets with large temperature contrasts have counterparts with gases flowing in tubes, which, together with the evident interest of these flows for heat exchangers, justify an analysis parallel to that carried out for jets in Refs. 8-10. Such an analysis is the purpose of this paper. Its distinctive feature, in comparison with the case of jets, is the presence of a pressure gradient along the tube, which is generated to ensure conservation of the mass flux. An ablation front appears in the tube when the inlet gas temperature is much smaller than the temperature of the wall, in which case the cold gas is rapidly squeezed into a thin core around the axis of the tube. No ablation front appears when the inlet gas temperature is much larger than the temperature of the wall.

\section{FORMULATION}

The following problem is considered for definiteness. A mass flux $G$ of a gas initially in a reservoir at temperature $T_{0}$ and density $\rho_{0}$ discharges through a circular tube of radius $a$ whose wall is kept at a temperature $T_{w}$. The Mach number $U_{0} / \sqrt{\gamma R_{g} T_{0}}$, where $U_{0}=G / \pi a^{2} \rho_{0}$ and $R_{g}$ and $\gamma$ are the gas constant and ratio of specific heats, is assumed to be small. The Reynolds number $R e=\rho_{0} U_{0} a / \mu_{0}$, where $\mu_{0}$ is the viscosity of the gas at temperature $T_{0}$, is assumed to be large. In these conditions, the quasi-isobaric and boundary layer approximations can be used. In addition, it is assumed that the Prandtl number of the gas is constant and its viscosity and thermal conductivity are given by the power laws $\mu / \mu_{0}=\lambda / \lambda_{0}=\left(T / T_{0}\right)^{\sigma}$, where $\lambda_{0}$ is the thermal conductivity at temperature $T_{0}$. Defining $\epsilon=T_{0} / T_{w}$ and scaling $T$ and $\rho$ with $T_{w}$ and $\epsilon \rho_{0}$, the distances along the tube and normal to its axis $\left(x\right.$ and $r$ ) with $\epsilon^{\sigma}$ Rea and $a$, the axial and radial components of the velocity ( $u$ and $v$ ) with $U_{0} / \epsilon$ and $U_{0} /\left(\epsilon^{\sigma+1} R e\right)$, and the pressure variations with $\rho_{0} U_{0}^{2} / \epsilon$, the 
equations and boundary conditions governing the flow in the tube, assumed to be laminar, are

$$
\begin{gathered}
\frac{\partial}{\partial x}(\rho u)+\frac{1}{r} \frac{\partial}{\partial r}(r \rho v)=0 \\
\rho u \frac{\partial u}{\partial x}+\rho v \frac{\partial u}{\partial r}=-\frac{\mathrm{d} p}{\mathrm{~d} x}+\frac{1}{r} \frac{\partial}{\partial r}\left(r T^{\sigma} \frac{\partial u}{\partial r}\right) \\
\rho u \frac{\partial T}{\partial x}+\rho v \frac{\partial T}{\partial r}=\frac{1}{\operatorname{Pr}} \frac{1}{r} \frac{\partial}{\partial r}\left(r T^{\sigma} \frac{\partial T}{\partial r}\right) \\
\rho T=1, \\
x=0: \quad u=\epsilon, \quad T=\epsilon, \quad p=0 \\
r=0: \quad \frac{\partial u}{\partial r}=\frac{\partial T}{\partial r}=v=0 \\
r=1: \quad u=v=T-1=0 .
\end{gathered}
$$

Equations (1)-(3) are the continuity, axial momentum, and energy conservation equations, and (4) is the equation of state in the quasi-isobaric approximation. Here $\operatorname{Pr}=\mu_{0} c_{p} / \lambda_{0}$, where $c_{p}$ is the specific heat at constant pressure and $p(x)$ is the pressure of the gas referred to the inlet pressure, which is uniform in each cross-section of the tube and must be found as part of the solution to satisfy the mass conservation condition $\int_{0}^{1} \rho u r \mathrm{~d} r=1 / 2$ [obtained by integrating Eq. (1) across the tube and using Eqs. (5)-(7)].

The solution of the problem must be computed numerically. For the numerical treatment, Eqs. (1)-(3) are discretized using Keller's second order finite difference method ${ }^{15}$ and solved with a standard iterative method.

\section{RESULTS AND DISCUSSION}

The purpose of this paper is to describe the structure of the solution of Eqs. (1)-(7) for very small and very large values of $\epsilon$, when the inlet temperature of the gas is very low or very high compared to the temperature of the wall. A numerical solution of the problem for $\epsilon=0.02, \sigma=0.75$, and $\operatorname{Pr}=1$ is displayed in Fig. 1. Some isotherms and streamlines of the flow are shown in the upper and lower halves of Fig. 1(a) together with temperature and velocity profiles at various sections of the tube, while Fig. 1(b) shows profiles of the radial heat flux and shear stress (upper and lower halves, respectively) and Fig. 1(c) shows the temperature and velocity along the axis of the tube. As can be seen, the cold gas rapidly confines itself to a thin core around the axis whose velocity increases with streamwise distance but remains small compared to the velocity of the hot gas filling most of the tube cross-section. The radius of the central core decreases along the tube, partially due to this acceleration but also because of the ablation due to radial conduction. The ablation continuously decreases the mass flux of cold gas until the central core disappears at about $x=0.5$, and the velocity and temperature in Fig. 1(c) increase smoothly downstream of this point toward the final state $u=2\left(1-r^{2}\right)$,
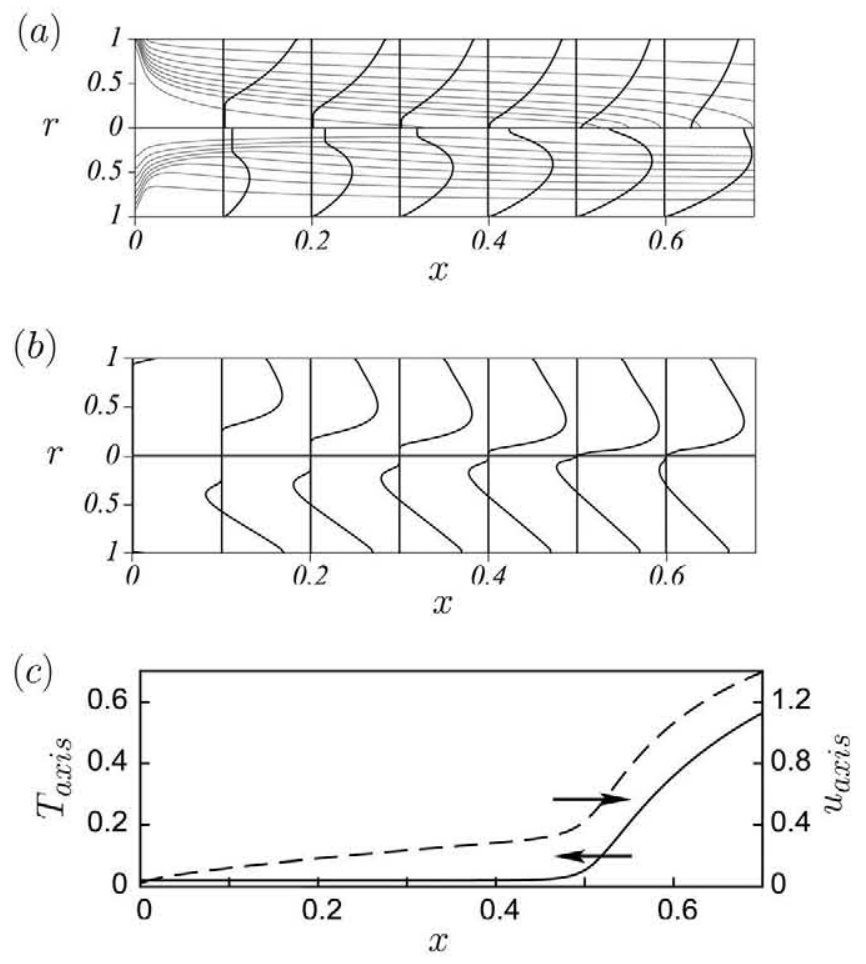

FIG. 1. Numerical solution of (1)-(7) for $\epsilon=0.02, \sigma=0.75$, and $P r=1$. (a) Isotherms and temperature profiles (upper half) and streamlines and velocity profiles (lower half). (b) Profiles of minus the radial heat flux $\left(\mathrm{Pr}^{-1} T^{\sigma} \partial T / \partial r\right.$, upper half) and the shear stress $\left(T^{\sigma} \partial u / \partial r\right.$, lower half). (c) Temperature (solid) and velocity (dashed) along the axis of the tube.

$T=1$. The length required for the ablation front to reach the axis and that required subsequently for the flow to reach uniform temperature are both of order unity with the scales selected. The existence of an ablation front that neatly bounds the cold gas in the asymptotic limit $\epsilon \rightarrow 0$ is a consequence of the variation of the thermal conductivity with temperature. As for the related problem of a jet of cold gas discharging into a hot stagnant gas analyzed by SánchezSanz et al., ${ }^{9,10}$ Fig. 1(b) shows that the shear stress and the heat flux scaled with their characteristic values in the hot gas [with $\left(\mu_{0} / \epsilon^{\sigma}\right)\left(U_{0} / a\right)$ and $\left(\lambda_{0} / \epsilon^{\sigma}\right)\left(T_{w} / a\right)$ ] tend to zero at the ablation front when $\epsilon \rightarrow 0$, leaving a mass flux of order unity (up to logarithms of $\epsilon$ ) that crosses the front and is subsequently heated up.

At variance with the case of a jet, the condition that the continuously increasing mass flux of hot gas must be confined to a tube of constant radius leads to the appearance of a pressure force $-\mathrm{d} p / \mathrm{d} x>0$ in Eq. (2). This pressure force also accelerates the cold gas, confining it to the thin core mentioned above. With the variables scaled as in Eqs. (1)-(7), it is $(\rho, T)=O(1)$ in the hot gas, where also $u=O(1)$ when the hot gas accounts for a substantial fraction of the total mass flux, for $x=O(1)$ and beyond. The pressure variations generated in the hot gas must be $p=O(1)$ in order for the pressure force to have an effect on this gas. In the core of cold gas, where $\rho=O\left(\epsilon^{-1}\right)$, these pressure variations lead to a dimensionless velocity $u_{c}=O\left(\epsilon^{1 / 2}\right)$, which is large compared to the inlet velocity $(u=\epsilon$ at $x=0)$ but still small compared to the velocity of the hot gas. By continuity, the radius of the core of cold gas is $r_{c}=O\left(\epsilon^{1 / 4}\right)$. Viscous forces and 
(a)

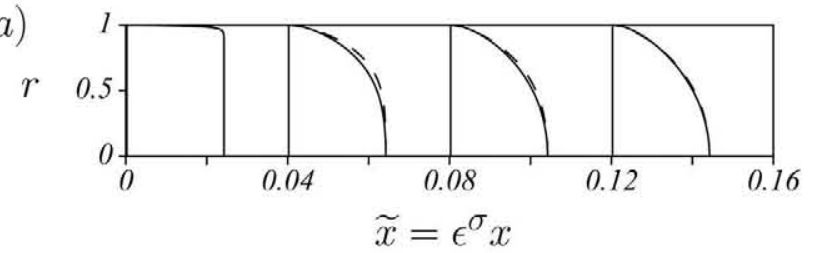

(b)

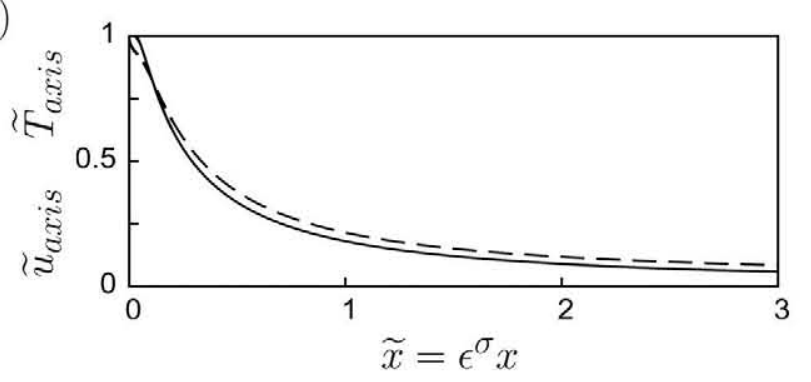

FIG. 2. Numerical solution of (1)-(7) for $\epsilon=50, \sigma=0.75$ and $P r=0.7$. (a) Profiles of temperature (solid) and velocity (dashed) at four sections near the inlet scaled with the values of these variables at the axis of the tube. (b) Rescaled temperature (solid) and velocity (dashed) along the axis of the tube.

heat conduction are negligible in the cold gas because $\left[r^{-1} \partial\left(r T^{\sigma} \partial u / \partial r\right) / \partial r\right] /(\rho \boldsymbol{v} \cdot \mathbf{V} u)=O\left(\epsilon^{\sigma}\right) \ll 1$ in Eq. (2) and a similar estimate holds for Eq. (3). Further analysis of the asymptotic limit $\epsilon \rightarrow 0$ is given in the appendix.

Consider now the solution of Eqs. (1)-(7) for large values of $\epsilon$, when the inlet temperature of the gas is large compared to the temperature of the wall. The dimensionless viscosity and conductivity of the hot gas are now large, of order $\epsilon^{\sigma}$, and therefore the characteristic dimensionless length of the region along the tube where convection and radial diffusion balance each other is small of order $\epsilon^{-\sigma}$. Equations (1)-(4) and (6) are left unchanged if $(x, r, u, v, p$, $\rho, T)$ are rescaled with the factors $\left(\epsilon^{-\sigma}, 1, \epsilon, \epsilon^{\sigma+1}, \epsilon, \epsilon^{-1}, \epsilon\right)$, respectively. Using tildes to denote rescaled variables, the inlet conditions (5) become $\tilde{u}=\tilde{T}=1, \tilde{p}=0$ at $\tilde{x}=0$, while the conditions (7) at the tube wall become $\tilde{u}=\tilde{v}=0$, $\tilde{T}=1 / \epsilon$ at $\tilde{r}=1$. This is the only place where $\epsilon$ appears in the rescaled problem, and the conditions at the wall reduce to $\tilde{u}=\tilde{v}=\tilde{T}=0$ at $\tilde{r}=1$ in the limit $\epsilon \rightarrow \infty$.

Figure 2 shows some temperature and velocity profiles computed for $\epsilon=50, \sigma=0.75, \operatorname{Pr}=0.7$, as well as the rescaled temperature and velocity along the axis of the tube. As can be seen, the flow evolves smoothly along the tube and the radial temperature and velocity profiles are smooth beyond the initial region of $\tilde{x} \ll 1$ where transport effects are confined to a thin boundary layer [leftmost profiles in Fig. 2(a)]. There is no ablation front for $\epsilon \gg 1$. The rate of cooling, as reflected by the evolution of the temperature on the axis in Fig. 2(b), continuously decreases along the tube due to the decrease of the dimensionless rescaled conductivity from values of $O(1)$ at the inlet to values of $O\left(\epsilon^{-\sigma}\right)$ in the last stages of cooling. The difference between temperature and velocity profiles, which are nearly identical in Fig. 2(a) for $\operatorname{Pr}=0.7$, increases when $\operatorname{Pr}$ is decreased, but this difference does not cause qualitative changes in the solution.

The viscosity and conductivity scaled with their values at the inlet tend to zero at the tube wall when $\epsilon \rightarrow \infty$, but the rescaled shear stress and heat flux, say $\tilde{\tau}_{w}$ and $\tilde{q}_{w}$, do not tend to zero. That $\operatorname{Pr}^{-1} \tilde{T}^{\sigma} \partial \tilde{T} / \partial \tilde{r} \rightarrow-\tilde{q}_{w}$ and $\tilde{T}^{\sigma} \partial \tilde{u} / \partial \tilde{r} \rightarrow-\tilde{\tau}_{w}$ for $\tilde{r} \rightarrow 1$ determine the local temperature and velocity as $\hat{T}=\left[(\sigma+1) \operatorname{Pr} \tilde{q}_{w}\right]^{1 /(\sigma+1)}(1-\tilde{r})^{1 /(\sigma+1)}$ and $\tilde{u}=(\sigma+1) \tilde{\tau}_{w}(1-\tilde{r})^{1 /(\sigma+1)} /\left[(\sigma+1) \operatorname{Pr} \tilde{q}_{w}\right]^{\sigma /(\sigma+1)}$ for $(1-\tilde{r})$ $\ll 1$ or, upon eliminating $\tilde{q}_{w}$ and $\tilde{\tau}_{w}$ between these relations and their derivatives, $\partial \tilde{T} / \partial \tilde{r}=-\tilde{T} /[(\sigma+1)(1-\tilde{r})]$ and $\partial \tilde{u} / \partial \tilde{r}=-\tilde{u} /[(\sigma+1)(1-\tilde{r})]$ for $(1-\tilde{r}) \ll 1$. These latter relations can be used as boundary conditions at a certain $\tilde{r}$ close to 1 to numerically solve the limiting problem for $\epsilon \rightarrow \infty$ in terms of the rescaled variables. The results of this computation differ very little from the results shown in Fig. 2 for $\epsilon=50$.

\section{CONCLUSIONS}

The laminar, low Mach number flow of a gas in a circular tube whose wall is kept at a constant temperature much larger or much smaller than the inlet temperature of the gas has been analyzed using the boundary layer and quasiisobaric approximations. In the limit when the inlet-to-wall temperature ratio tends to zero, pressure forces accelerate the cold gas and confine it to a thin core around the axis of the tube. An ablation front, where the heat flux tends to zero, bounds the cold gas and consumes it at a finite distance from the inlet. No ablation front appears in the opposite limit when the inlet-to-wall temperature ratio tends to infinite, in which the shear stress and the heat flux are different from zero at the wall despite the fact that the viscosity and the thermal conductivity of the gas tend to zero there.

\section{ACKNOWLEDGMENTS}

This work was supported through projects DPI201020450-C03-01 and CSD2010-00010 (Ministerio de Ciencia e Innovación) and S2009/ENE-1597 (Comunidad de Madrid).

\section{APPENDIX: LIMIT $\epsilon \rightarrow 0$}

The temperature and velocity in the hot gas near the ablation front, for $0<r-r_{c} \ll r_{c}$, can be determined as in Refs. 5 and 6. For $\sigma>1 / 2$ and $\sigma \operatorname{Pr} \neq 1$, these variables are

$$
\begin{aligned}
u & =\frac{r_{c}}{(\sigma P r-1) \phi} \frac{\mathrm{d} p}{\mathrm{~d} x}\left(r-r_{c}\right)+a\left(r-r_{c}\right)^{1 / \sigma P r}+\cdots, \\
\rho v & =\frac{\phi}{r_{c}}+\cdots, \quad \frac{1}{\rho}=T=\left(\sigma \operatorname{Pr} \frac{\phi}{r_{c}}\right)^{1 / \sigma}\left(r-r_{c}\right)^{1 / \sigma}+\cdots,
\end{aligned}
$$

where $\phi(x)$ and $a(x)$ are functions of $x$ to be determined from the numerical solution together with $r_{c}(x)$ and the pressure gradient. In particular, $2 \pi \phi$ is the mass flux crossing the ablation front per unit length along the tube, which is given by

$$
\phi=-\frac{1}{2} \frac{\mathrm{d}}{\mathrm{d} x}\left(\sqrt{-\frac{2 p}{\epsilon}} r_{c}^{2}\right),
$$

from the continuity equation for the core of cold gas where Bernouilli's equation $u_{c}^{2} / 2 \epsilon+p=0$ has been used to eliminate $u_{c}$. The shear stress and heat flux computed from 
Eq. (A1) tend to zero when $r \rightarrow r_{c}$. Since $r_{c}=O\left(\epsilon^{1 / 4}\right) \ll 1$, the range of $r$ where Eq. (A1) can be used is very narrow in the present problem. However, the analysis leading to Eq. (A1) can be easily extended to take into account the curvature of the ablation front. The results valid for any $\left(r-r_{c}\right) \ll 1$ are

$$
\begin{aligned}
u= & \frac{r_{c}}{(\sigma P r-1) \phi} \frac{\mathrm{d} p}{\mathrm{~d} x}\left(r-r_{c}\right) F_{1}\left(\frac{r}{r_{c}}\right) \\
& +\left[a+\frac{r_{c}^{2-1 / \sigma P r}}{2 \sigma P r \phi} \frac{\mathrm{d} p}{\mathrm{~d} x} F_{2}\left(\frac{r}{r_{c}}\right)\right]\left(r-r_{c}\right)^{1 / \sigma P r} F_{1}\left(\frac{r}{r_{c}}\right)+\cdots, \\
\rho v r= & \phi+\cdots, \\
T= & (\sigma \operatorname{Pr} \phi)^{1 / \sigma}\left(\ln \frac{r}{r_{c}}\right)^{1 / \sigma}+\cdots,
\end{aligned}
$$

with

$$
\begin{aligned}
& F_{1}(\xi)=\exp \left(\frac{1}{\sigma P r} \int_{1}^{\xi} \frac{\xi-1-\xi \ln \xi}{\xi(\xi-1) \ln \xi} \mathrm{d} \xi\right), \\
& F_{2}(\xi)=\int_{1}^{\xi} \frac{\xi^{2}-1-2 \xi \ln \xi F_{1}(\xi)}{\xi(\xi-1)^{1 / \sigma P r} \ln \xi F_{1}(\xi)} \mathrm{d} \xi .
\end{aligned}
$$

In principle, these results could be used to set up a limiting problem for $\epsilon \rightarrow 0$ involving the hot gas only. In fact, the core of cold gas acts as a porous solid rod of radius $r_{c}(x)$ through which the mass flux (A2) is injected with $u=T=0$. At first sight, Eqs. (1)-(4) with these boundary conditions and boundary conditions (7) at the tube wall should have a solution [determining in particular $p(x)$ ] for any $r_{c}(x)$. However, the heat flux reaching the rod per unit length of the tube, $q(x)$ say, will not be zero in general, leading to a temperature of the form $T=[(\sigma+1) \operatorname{Pr} q / 2 \pi]^{1 /(\sigma+1)}\left(\ln r / r_{c}\right)^{1 /(\sigma+1)}$ for $r \ll 1$. The additional condition that $q(x)=0$, so that the temperature takes the form (A3) near the rod, determines $r_{c}(x)$. Unfortunately the limiting problem for $\epsilon \ll 1$ is not free of the small parameter $\epsilon$ due to the presence of logarithms in Eq. (A3), and thus it is of limited practical value. The analogous limiting problem for a two-dimensional channel, with effectively a thin porous layer around its center-plane, does not contain $\epsilon$ and may be more useful.

${ }^{1}$ W. M. Kays and W. B. Nicoll, "Laminar flow heat transfer to a gas with large temperature differences," ASME J. Heat Transfer 85, 329 (1963).

${ }^{2}$ M. E. Davenport and G. Leppert, "The effect of transverse temperature gradients on the heat transfer and friction for laminar flow of gases," ASME J. Heat Transfer 87, 191 (1965).

${ }^{3}$ P. M. Worsoe-Schmidt and G. Leppert, "Heat transfer and friction for laminar flow of a gas in a circular tube at a high heating rate," Int. J. Heat Mass Transfer 8, 1291 (1965).

${ }^{4} \mathrm{P}$. M. Worsoe-Schmidt, "Heat transfer and friction for laminar flow of helium and carbon dioxide in a circular tube at a high heating rate," Int. J. Heat Mass Transfer 9, 1291 (1966).

${ }^{5}$ F. J. Higuera and A. Liñán, "Flow field of a diffusion flame attached to a thick-walled injector between two coflowing reactant streams," J. Fluid Mech. 329, 389 (1996).

${ }^{6}$ F. J. Higuera, "Boundary layer separation due to gas thermal expansion," Phys. Fluids 9, 2841 (1997).

${ }^{7}$ M. Sánchez-Sanz, "Laminar gas jets with extreme gas-to-ambient density ratios," Ph.D. dissertation, University Carlos III de Madrid, 2007.

${ }^{8}$ M. Sánchez-Sanz, A. L. Sánchez, and A. Liñán, "Fronts in hightemperature laminar gas jets," J. Fluid Mech. 547, 257 (2006).

${ }^{9}$ M. Sánchez-Sanz, A. L. Sánchez, and A. Liñán, in New Research Trends in Fluid Mechanics: Proceedings of the Fifth International Conference on Fluid Mechanics, Shanghai, China, 15-19 August 2007, edited by F. G. Zhuang and J. C. Li (Tsinghua University Press/Springer, New York, 2007).

${ }^{10} \mathrm{M}$. Sánchez-Sanz, A. L. Sánchez, and A. Liñán, "Variable-density jet flows induced by concentrated sources of momentum and energy," Theor. Comput. Fluid Dyn. 25, 281 (2011).

${ }^{11}$ Ya. B. Zeldovich and A. S. Kompaneetz, in Collection of Papers Dedicated to the 70th Birthday of Academician A. F. Ioffe [Izd. Akad. Nauk SSSR], Moscow, 1950, pp. 61-71.

${ }^{12} \mathrm{~F}$. A. Williams, "On the assumptions underlying droplet vaporization and combustion theories," J. Chem. Phys. 33, 133 (1960).

${ }^{13}$ C. S. Tarifa, A. Crespo, and E. Fraga, "A theoretical model for the combustion of droplets in super-critical conditions and gas pockets," Astron. Acta 17, 685 (1972).

${ }^{14} \mathrm{~A}$. Crespo and A. Liñán, "Unsteady effects in droplet evaporation and combustion," Combust. Sci. Technol. 11, 9 (1975).

${ }^{15} \mathrm{H}$. B. Keller, "Numerical methods in boundary layer theory," Annu. Rev. Fluid Mech. 10, 417 (1978). 